\title{
STUDY OF DROPLET ACTIVATION IN THIN CLOUDS USING GROUND-BASED RAMAN LIDAR AND ANCILLARY REMOTE SENSORS
}

\author{
Marco Rosoldi $^{1 *}$, Fabio Madonna ${ }^{1}$, Pilar Gumà Claramunt ${ }^{1}$ and Gelsomina Pappalardo ${ }^{1}$ \\ ${ }^{1}$ Consiglio Nazionale delle Ricerche (CNR), Istituto di Metodologie per l'Analisi Ambientale (IMAA), \\ C.da S. Loia, I-85050, Tito Scalo, Potenza, Italy, *Email: marco.rosoldi@imaa.cnr.it
}

\begin{abstract}
A methodology for the study of cloud droplet activation based on the measurements performed with ground-based multi-wavelength Raman lidars and ancillary remote sensors collected at CNR-IMAA observatory, Potenza, South Italy, is presented. The study is focused on the observation of thin warm clouds. Thin clouds are often also optically thin: this allows the cloud top detection and the full profiling of cloud layers using ground-based Raman lidar. Moreover, broken clouds are inspected to take advantage of their discontinuous structure in order to study the variability of optical properties and water vapor content in the transition from cloudy regions to cloudless regions close to the cloud boundaries. A statistical study of this variability leads to identify threshold values for the optical properties, enabling the discrimination between clouds and cloudless regions. These values can be used to evaluate and improve parameterizations of droplet activation within numerical models. A statistical study of the co-located Doppler radar moments allows to retrieve droplet size and vertical velocities close to the cloud base. First evidences of a correlation between droplet vertical velocities measured at the cloud base and the aerosol effective radius observed in the cloud-free regions of the broken clouds are found.
\end{abstract}

\section{INTRODUCTION}

Studies on global climate change show that the effects of aerosol-cloud interactions (ACI) on the Earth's climate and radiative balance, also known as indirect aerosol effects, are the most uncertain among all the effects involving the atmospheric constituents and processes [1]. The activation of cloud droplets occurs through the water vapor condensation when super-saturation conditions are reached in an air mass. It represents the microphysical link between aerosols and clouds and it is the basis towards a better understanding of ACI.

The droplet activation and the ACI are not yet accurately quantified and parameterized in the weather and climate numerical models due to the complexity of the processes which take place far away from the ground, where the availability of accurate in situ measurements is limited in time and space sampling. Therefore, the study of droplet activation and ACI is strongly needed to evaluate and improve the ability of numerical models to forecast clouds and their microphysical properties, as well as to better quantify ACI in global climate models. This investigation needs an accurate estimation of optical and microphysical properties of clouds and aerosols close to the cloud boundaries, that can be achieved using ground-based remote sensing techniques.

This work describes a methodology to improve the knowledge of the processes underlying the droplet activation. It is based on the observations performed with multi-wavelength $(355,532$ and 1064 nm) Raman lidars, a Ka-band cloud Doppler radar and a microwave radiometer operative at CIAO (CNR-IMAA Atmospheric Observatory), located in Potenza, Southern Italy $(40.60 \mathrm{~N}$, 15.72E, $760 \mathrm{~m}$ above sea level) and on the study of thin warm clouds. These clouds are low or midlevel super-cooled clouds characterized by a liquid water path (LWP) less than about $100 \mathrm{gm}^{-2}$ [2]. Thin warm clouds are often optically thin, i.e they allow the Raman lidar to measure the backscattered signal through the entire cloud structure and above, enabling the cloud top detection. Optically thin broken warm clouds are observed, where broken refers to single layer clouds with a not homogeneous horizontal structure, characterized by cloud fields separated by, cloud-free regions. The discontinuous structure of broken clouds allows to study the 
variability of optical properties and water vapor content in the transition from cloudy regions to cloudless regions close to cloud boundaries.

\section{METHODOLOGY}

Night time observations of low and mid-level optically thin broken clouds are performed simultaneously with the multi-wavelength Raman lidars, the cloud Doppler radar and the microwave radiometer. The cloud phase is identified using the radar linear depolarization ratio (LDR) and atmospheric temperature profiles obtained by the microwave radiometer in the time range when the cloud is observed.

Taking advantage of the discontinuous structure of the broken clouds, the vertical profiles of the extensive aerosol optical properties (extinction coefficient $\alpha$ at 355 and $532 \mathrm{~nm}$ and backscattering coefficients $\beta$ at 355, 532 and 1064 $\mathrm{nm}$ ) and of the water vapor content (water vapor mixing ratio and relative humidity) are retrieved using the combined Raman-elastic lidar technique in two different ways: "with clouds", by averaging only the lidar signals containing clouds, in a selected time window; "without clouds", by averaging only the cloud-free signals before and after the clouds (skipping the signals containing clouds), in the same time window. The lidar signals contaminated by clouds are identified by visual inspection of the nitrogen Raman lidar signals at $387 \mathrm{~nm}$, assuming that a strong negative slope in these signals is due to the extinction by liquid water droplets. The profiles with clouds provide the cloud optical properties and the water vapor content in cloudy regions, while the profiles without clouds provide the aerosol optical properties and water vapor content in cloudless regions close to the cloud boundaries.

For each observed cloud layer, the vertical profiles of optical properties and water vapor content are calculated using the same effective vertical resolution. The selected time windows for lidar analysis are in the range 15 - 45 minutes, while the used effective vertical resolutions are in the range 160-350 $\mathrm{m}$. These resolutions have been optimized in a way to obtain lidar profiles at the highest vertical resolution with a random uncertainty lower than $30 \%$. The profiles of extinction coefficient with clouds are corrected for multiple scattering $[3,4]$.
For each observed cloud, the vertical regions where extinction coefficients at $355 \mathrm{~nm}$ with and without clouds do not show significant differences, within their error bars, represent atmospheric regions out of the cloud layer, where aerosols are not activated into droplets; the altitude range where the extinction coefficient at $355 \mathrm{~nm}$ with clouds is significantly higher than the corresponding extinction coefficient without clouds constitutes the region where aerosol particles grow and droplet activation occurs, identified as the "activation region".

The cloud base and top are determined by using the nitrogen Raman lidar signals at $387 \mathrm{~nm}$ with and without clouds, calibrated on the same molecular lidar signal at $387 \mathrm{~nm}$ derived from a standard atmosphere model. The cloud base height is identified by an inflection point in the Raman signal with clouds, corresponding to a local minimum of the first derivative of the signal; specifically it is identified as the height at which the derivative starts to decrease on the decreasing edge of the local minimum. The cloud top height is identified as the height where the Raman signal with clouds starts to overlap the corresponding Raman signal without clouds.

The cloud base and cloud top temperatures as well as cloud the LWP are estimated by averaging the microwave radiometer products in the same time window of the lidar analysis.

The cloud optical depth (COD) is obtained by integrating the extinction coefficient profile with clouds, corrected for multiple scattering, over the altitude range between the base and the top of the cloud.

The source and type of aerosols in cloud-free regions close to the cloud boundaries are identified from the analysis of the air mass backtrajectories provided by NOAA Hysplit model [5], along with the values of multi-wavelength aerosol lidar ratios (S) and the Ångström exponents (å) [6], averaged over the altitude range between the cloud base and the cloud top. In the same region, the aerosol size distribution close to the clouds is also retrieved from multi-wavelength aerosol extensive optical properties, averaged over the altitude range between the base and the top of the cloudy region, using the algorithm developed by Veselovskii [7]. 


\section{RESULTS}

Twenty cloud layers have been studied. Using the methodology described in section 2 , it is found that these clouds are characterized by a vertical extension of the activation region in the range 290-1040 m, the cloud base height in the range 1440-4550 $\mathrm{m}$ above sea level (a.s.1.), the cloud top height in the range $1640-4730 \mathrm{~m}$ a.s.l., the cloud thickness in the range $90-500 \mathrm{~m}$, the cloud base and the cloud top temperatures in the range 270$286 \mathrm{~K}$ and $268-284 \mathrm{~K}$, respectively, the COD at $355 \mathrm{~nm}$ in the range 0.06-1.20. All the observed clouds are liquid or mixed phase clouds with a LWP lower than $180 \mathrm{gm}^{-2}$. In the cloud-free regions close to the cloud boundaries, the aerosol type is originated from different sources (dust, continental, urban-industrial, marine, mixed).

Figure 1 shows the probability density function (pdf) of $\alpha$ (panel a), $\beta$ (panel b) and S (panel c) at $355 \mathrm{~nm}$, calculated from profiles with clouds (blue columns) and without clouds (red columns) in the altitude range between the base and the top of the activation region. These pdfs lead to identify threshold values enabling the discrimination between clouds and cloud-free atmosphere. In particular, values of $\alpha$ and $\beta$ higher than $3.5 \times 10^{-4}$ $\mathrm{m}^{-1}$ and $1.0 \times 10^{-5} \mathrm{~m}^{-1} \mathrm{sr}^{-1}$, respectively, and values of S lower than 25 sr (blue areas in fig.1) show a very high probability of droplet activation and cloud formation and are classified as cloudy regions. Values of $\alpha$ and $\beta$ lower than $1.0 \times 10^{-4}$ $\mathrm{m}^{-1}$ and $0.6 \times 10^{-5} \mathrm{~m}^{-1} \mathrm{sr}^{-1}$, respectively, and values of $\mathrm{S}$ higher than $55 \mathrm{sr}$ (red areas in fig.1) show a very low probability of droplet activation and cloud formation and are classified as cloudless regions. Values of $\alpha, \beta$, and $\mathrm{S}$ between the above threshold values, i.e. $\alpha$ in the range $1.0 \times 10^{-4}-3.5$ $\times 10^{-4} \mathrm{~m}^{-1}, \beta$ in the range $0.6 \times 10^{-5}-1.0 \times 10^{-5} \mathrm{~m}^{-1}$ $\mathrm{sr}^{-1}$ and $\mathrm{S}$ in the range $25-55 \mathrm{sr}$ (green areas in fig.1) show comparable probabilities of droplet activation or not; these values do not allow to discriminate between cloudy and cloudless regions and might correspond to the transition region between clouds and cloud-free atmosphere also called "Twilight Zone" [8]. These results can be used to directly evaluate and improve the ability of cloud numerical models to predict the formation of liquid water clouds, by ground-based Raman lidar measurements of extinction and backscattering coefficients. This evaluation also implies an indirect validation of parameterizations used in numerical models for the droplet activation.
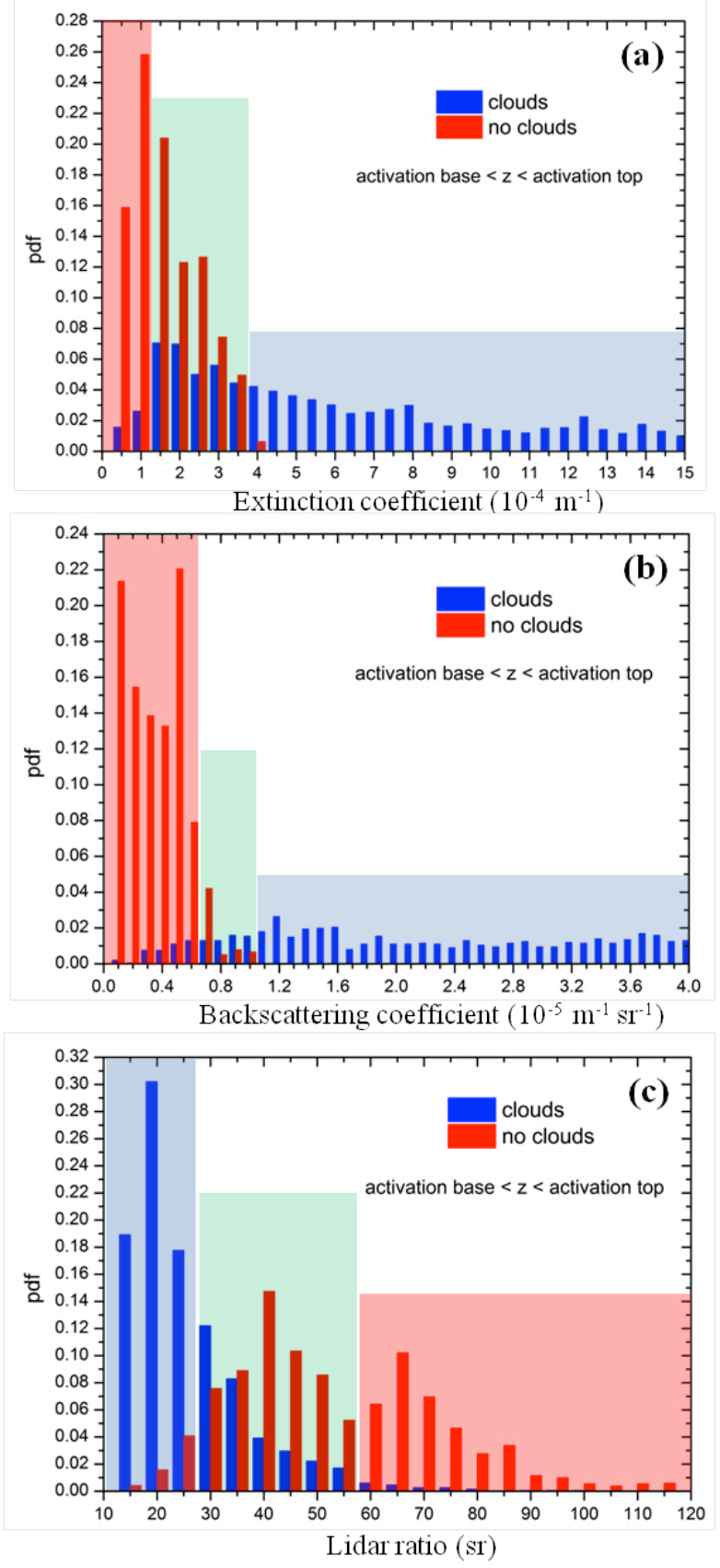

Figure 1. Probability density function (pdf) of the aerosol extinction coefficient (a), aerosol backscatter coefficient (b) and lidar ratio (c) at $355 \mathrm{~nm}$, calculated from the Raman lidar profiles "with clouds" (blue columns) and "without clouds" (red columns) in the altitude ranges between the base and the top of the activation region.

Only in ten of the considered cases the radar provides a Doppler spectrum. The pdfs of mean Doppler velocity and reflectivity measured by the 
radar at the closest available altitude to the cloud base, are calculated. The Doppler velocity pdf shows that close to the cloud base droplet vertical velocity is typically dominated by downdrafts, with velocities ranging within $-1.5 \mathrm{~m} \mathrm{~s}^{-1}$ and + $0.5 \mathrm{~m} \mathrm{~s}^{-1}$, with a median value around $-0.25 \mathrm{~m} \mathrm{~s}^{-1}$. Using the radar reflectivity and scattering simulations [9], it is possible to relate the pdf of the radar reflectivity to droplet size: the two main modes of the pdf are corresponding to droplet sizes around $10 \mu \mathrm{m}$ and $15 \mu \mathrm{m}$, respectively. Finally, the scatter plot of droplet vertical velocities, measured at the cloud base and averaged over the time window of lidar analysis for each cloud layer, versus the effective radius of aerosol particles in cloud-free regions close to the cloud boundaries is considered. The scatter plot indicates that aerosols with an effective radius smaller than $0.8 \mu \mathrm{m}$ are activated to form droplets moving downward, while aerosols with larger effective radius tend to form droplets moving upward.

\section{CONCLUSIONS}

A methodology for the experimental investigation of cloud droplet activation is presented. The study is focused on optically thin warm clouds with a broken structure. It is based on the observations performed with ground-based multi-wavelength Raman lidars, a cloud Doppler radar and a microwave radiometer. Geometrical and microphysical properties for twenty cases characterized by this cloud type are reported. Type, source, and microphysical properties of aerosols in cloud-free regions close to the cloud boundaries are also retrieved. To enable an efficient discrimination between clouds and cloudless regions, threshold values of $\alpha, \beta$ and $\mathrm{S}$ at $355 \mathrm{~nm}$ are identified. These values can be used to evaluate and improve numerical models and their parameterizations of cloud droplet activation. Finally, droplet sizes and vertical velocities close to the cloud base have been correlated with the size of aerosol particles, revealing that droplet activation for aerosol particles smaller than 0.8 $\mu \mathrm{m}$ generates strong downdrafts, while activation for particles larger than $0.8 \mu \mathrm{m}$ generates moderate updrafts.

\section{ACKNOWLEDGEMENT}

This work was supported by the European Union (FP7/2007-2013) for the ACTRIS Research Infrastructure under grant n. 262254.

\section{REFERENCES}

[1] Stocker, T.F. et al., 2013: Contribution of Working Group I to the Fifth Assessment Report of the Intergovernmental Panel on Climate Change, Cambridge University Press.

[2] Turner, D. D. et al., 2007: Thin liquid water clouds: Their importance and our challenge, Bull. Amer. Meteor. Soc., 88, 177-190.

[3] Eloranta, E.E., 1998: Practical model for the calculation of multiply scattered lidar returns, Applied Optics, Vol.37, N.12, 2464-2472.

[4]Wandinger, U., 1998: Multiple scattering influence on extinction and backscatter coefficient measurements with Raman and high spectral resolution lidars, Applied Optics, Vol.37, N.3, 417-427.

[5] Draxler, R.R., G.D. Rolph, 2014: HYSPLIT (Hybrid Single-Particle Lagrangian Integrated Trajectory) Model access via NOAA ARL READY Website, NOAA Air Resources Laboratory, Silver Spring, MD.

[6]Müller, D. et al., 2007: Aerosol-typedependent lidar ratios observed with Raman lidar, Journal of Geophysical Research: Atmospheres, Vol.112, D16202.

[7] Veselovskii, I. et al., 2004: Inversion of multi wavelength Raman lidar data for retrieval of bimodal aerosol size distribution, Applied Optics, Vol.43, N.5, $1180-1195$.

[8] Koren, I. et al., 2007: On the twilight zone between clouds and aerosols, Geophysical Research Letters, Vol. 34, L08805.

[9] Carey, L. D., S. A. Rutledge, 1998: Electrical and multiparameter radar observations of a severe hailstorm, J. Geophys. Res., 103, 13979-14000. 